\title{
3C 286: a bright, compact, stable, and highly polarized calibrator for millimeter-wavelength observations
}

\author{
I. Agudo ${ }^{1,2}$, C. Thum ${ }^{3}$, H. Wiesemeyer ${ }^{4,5}$, S. N. Molina ${ }^{1}$, C. Casadio ${ }^{1}$, J. L. Gómez ${ }^{1}$, and D. Emmanoulopoulos ${ }^{6}$ \\ 1 Instituto de Astrofísica de Andalucía, CSIC, Apartado 3004, 18080 Granada, Spain \\ e-mail: iagudo@iaa.es \\ 2 Institute for Astrophysical Research, Boston University, 725 Commonwealth Avenue, Boston, MA 02215, USA \\ 3 Institut de Radio Astronomie Millimétrique, 300 rue de la Piscine, 38406 St. Martin d'Hères, France \\ ${ }^{4}$ Max-Planck-Institut für Radioastronomie, Auf dem Hügel 69, 53121 Bonn, Germany \\ 5 Instituto de Radio Astronomía Milimétrica, Avenida Divina Pastora 7, Local 20, 18012 Granada, Spain \\ ${ }^{6}$ School of Physics and Astronomy, University of Southampton, Southampton SO17 1BJ, UK
}

Received 10 January 2012 / Accepted 29 February 2012

\begin{abstract}
Context. Several millimeter and submillimeter facilities with linear polarization observing capabilities have started operating during the last years. These facilities, as well as other previous millimeter telescopes and interferometers, require bright and stable linear polarization calibrators to calibrate new instruments and to monitor their instrumental polarization. The current limited number of adequate calibrators implies difficulties in the acquisition of these calibration observations.

Aims. Looking for additional linear polarization calibrators in the millimeter spectral range, we started monitoring $3 \mathrm{C} 286$ in mid2006. This source is a standard and highly stable polarization calibrator for radio observations.

Methods. Here we present the $3 \mathrm{~mm}$ and $1 \mathrm{~mm}$ monitoring observations obtained between September 2006 and January 2012 with the XPOL polarimeter on the IRAM $30 \mathrm{~m}$ Millimeter Telescope.

Results. Our observations show that $3 \mathrm{C} 286$ is a bright source of constant total flux with $3 \mathrm{~mm}$ flux density $S_{3 \mathrm{~mm}}=(0.91 \pm 0.02) \mathrm{Jy}$. The $3 \mathrm{~mm}$ linear polarization degree $\left(p_{3 \mathrm{~mm}}=[13.5 \pm 0.3] \%\right)$ and polarization angle $\left(\chi_{3 \mathrm{~mm}}=[37.3 \pm 0.8]^{\circ}\right.$, expressed in the equatorial coordinate system) were also constant during the time span of our observations. Although with poorer time sampling and signal-tonoise ratio, our $1 \mathrm{~mm}$ observations of 3C 286 are also reproduced by a constant source of $1 \mathrm{~mm}$ flux density $\left(S_{1 \mathrm{~mm}}=[0.30 \pm 0.03] \mathrm{Jy}\right)$, polarization fraction $\left(p_{1 \mathrm{~mm}}=[14.4 \pm 1.8] \%\right)$, and polarization angle $\left(\chi_{1 \mathrm{~mm}}=[33.1 \pm 5.7]^{\circ}\right)$.

Conclusions. This, together with the previously known compact structure of $3 \mathrm{C} 286$ - extended by $\sim 3.5^{\prime \prime}$ in the sky - allow us to propose 3C 286 as a new calibrator for both single-dish and interferometric polarization observations at $3 \mathrm{~mm}$, and possibly at shorter wavelengths.
\end{abstract}

Key words. polarization - instrumentation: polarimeters - techniques: polarimetric - quasars: individual: 3C 286 submillimeter: general - radio continuum: general

\section{Introduction}

Despite the recent start of operations of quite a few millimeter observing facilities with the ability for high-precision polarization measurements (e.g., ALMA, Planck, the IRAM $30 \mathrm{~m}$ Telescope, SMA, KVN, BOOMERANG, CBI, MAXIPOL, QUaD, WMAP, and BICEP), the number of suitable polarization calibrators is still limited. The most commonly used sources for calibrating millimeter polarization observations are the Crab nebula (Taurus A, Aumont et al. 2010), Centaurus A (Zemcov et al. 2010), the limb of the Moon (Barvainis et al. 1988; Thum et al. 2003), and the diffuse Galactic emission (Matsumura et al. 2010). An ideal linearly polarized calibrator is a point-like, bright, and constant source of high linear-polarization fraction with a clearly defined and constant polarization angle. For most facilities designed to study the polarization properties of the cosmic microwave background, which still do not aim at observations with angular resolutions much better than $\sim 5^{\prime}$, one or more among the four calibrators mentioned above fulfill most of these conditions. However, the relatively broad angular extension of these four polarization calibrators $\left(z 5^{\prime}\right.$, where $5^{\prime}$ is the approximate angular extension of the Crab nebula at millimeter wavelengths; Aumont et al. 2010) may hamper polarization calibration observations at angular resolutions better than that. Even when this is not the case, the poor coverage of adequate calibrators in the sky often reveals the need for new polarized sources that are suitable for calibration.

We present the results from series of millimeter measurements with the IRAM $30 \mathrm{~m}$ Telescope to characterize 3C 286 (with $\mathrm{J} 2000$ equatorial coordinates: $\alpha=13^{\mathrm{h}} 31^{\mathrm{m}} 8.3^{\mathrm{s}}, \delta=$ $\left.+30^{\circ} 30^{\prime} 33^{\prime \prime}\right)$ as a new bright, compact, stable, and highly polarized calibrator for short millimeter observations. This source has been widely used for calibration at centimeter radio wavelengths both for total flux (Baars et al. 1977; Ott et al. 1994; Kraus et al. 1999) and linear polarization observations (Perley 1982; McKinnon 1992; Taylor et al. 2001; Gómez et al. 2002).

3C 286 is a bright, compact, steep-spectrum radio quasar (Peacock \& Wall 1982; Fanti et al. 1985) at a redshift $z=0.849$ (Burbidge \& Burbidge 1969). Radio imaging with the Very Large Array at subarcsecond resolution has revealed an extended structure composed of three misaligned bright features (e.g., Akujor \& Garrington 1995). Among these three structures, the second one in brightness is located 2.6" west-southwest from the brightest one. They are linked by a nearly straight jet-like bridge 


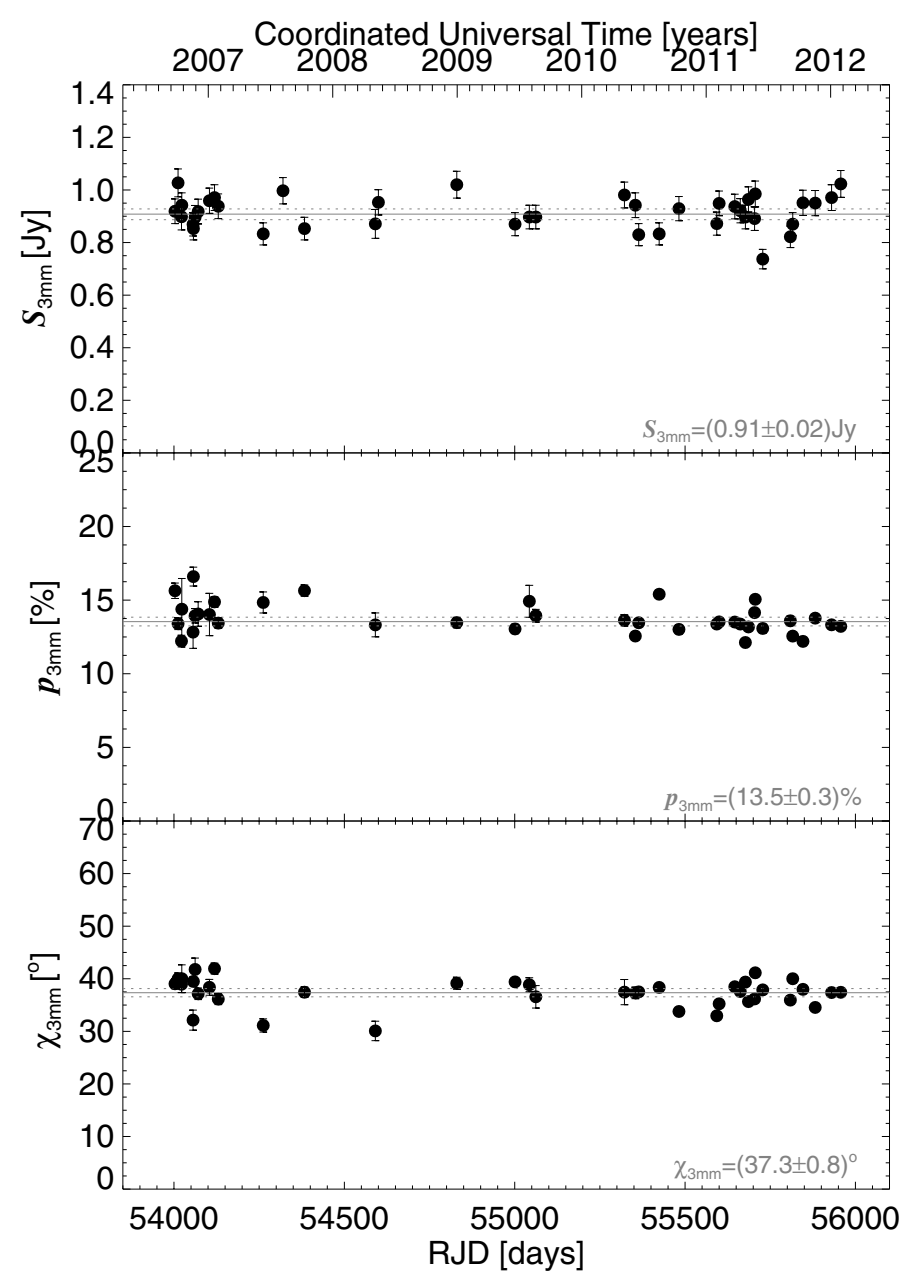

Fig. 1. $3 \mathrm{~mm}$ total flux and polarization evolution of $3 \mathrm{C} 286$. The fitted constant values of $S_{3 \mathrm{~mm}}, p_{3 \mathrm{~mm}}$, and $\chi_{3 \mathrm{~mm}}$ to be used for calibration through observations of $3 \mathrm{C} 286$, and their $95 \%$ confidence interval (obtained in Sect. 3.1), are given on every panel. The horizontal continuous and dotted lines symbolize this constant and 95\% interval, respectively.

of radio emission seen in high dynamic range images. The third emission region is located at $0.8^{\prime \prime}$ east from the brightest one. The two brightest emission features dominate the linear polarization emission and show their electric vector oriented nearly parallel to the axis of the jet-like structure between them. The integrated electric vector polarization angle of the source $^{1} \chi \approx 33^{\circ}$ at all observing wavelengths from $20 \mathrm{~cm}$ to $7 \mathrm{~mm}$ (Perley 1982; Taylor et al. 2001) $)^{2}$. The stability of $\chi$ along radio frequencies has also been demonstrated through polarization angle rotation measure studies (Rudnick \& Jones 1983). Very long baseline interferometric radio observations at milliarcsecond resolution of the brighter and more compact feature have revealed a clearly defined jet structure initially oriented in the southwest direction up to scales $>60$ milliarcsec, with the linear polarization fraction ranging from $p \approx 1 \%$ and $p \approx 20 \%$ and $\chi$ oriented nearly (but not exactly) parallel to the local jet axis (Jiang et al. 1996). These properties are not uncommon in radio-loud quasar jets. However, 3C 286 lacks the highly variable, low polarization and high brightness temperature $\left(T_{\mathrm{b}} \gtrsim 10^{10}-10^{11} \mathrm{~K}\right)$ radio core (Cotton et al. 1997) typical for radio quasars. This makes

\footnotetext{
1 To express polarization angles, we follow the IAU convention throughout. Such convention counts east from north in the equatorial coordinate system.

${ }^{2}$ See also http://www.vla.nrao.edu/astro/calib/polar/
}

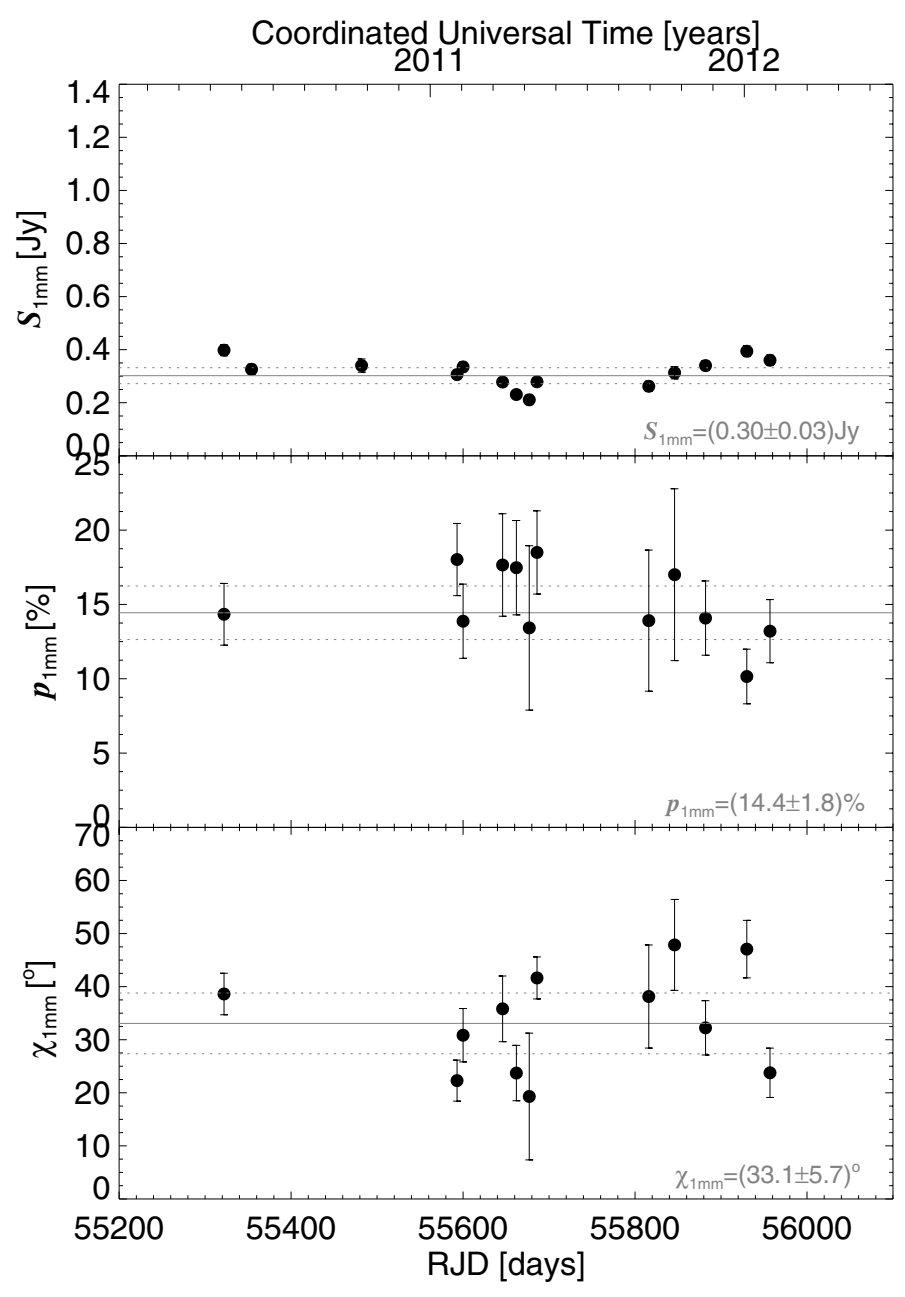

Fig. 2. Same as Fig. 1 but for the $1 \mathrm{~mm}$ data.

3C 286 an excellent total flux and polarization calibrator at radio wavelengths, and provides it with its characteristic non-variable and high integrated linear polarization degree $(p \approx 11 \%)$ at radio frequencies ${ }^{3}$. The lack of a typical radio core in 3C 286 was interpreted by Cotton et al. (1997) to be produced by strong relativistic de-boosting of the emission from the core when beamed in a direction significantly different from that of the observer's line of sight.

\section{Observations and data reduction}

The observations presented here were performed with the XPOL polarimeter (Thum et al. 2008) on the IRAM $30 \mathrm{~m}$ Telescope. The 3C 286 measurements were taken for calibration purposes under the POLAMI (POLarimetric AGN Monitoring at the IRAM-30 m-telescope) and the MAPI (Monitoring of AGN with Polarimetry at the IRAM-30 m-telescope) programs (e.g., Agudo et al. 2011b), and for a densely time-sampled $3 \mathrm{~mm}$ polarimetric monitoring program of blazar OJ287 (Agudo et al. 2011a). The time range of 3C 286 observations covers from 24 September, $2006(\mathrm{RJD}=54003)^{4}$ to 30 January, 2012 (RJD = 55 957); see Figs. 1 and 2, and Tables 1 and 2.

The standard XPOL set-up and calibration scheme introduced in Thum et al. (2008) and Agudo et al. (2010) were used.

\footnotetext{
3 http://www.vla.nrao.edu/astro/calib/polar/

4 RJD, i.e. reduced Julian date $=$ Julian date -240000 days.
} 
Table 1. $3 \mathrm{~mm}$ photo-polarimetric data from 3C 286.

\begin{tabular}{|c|c|c|c|c|}
\hline Date & $\begin{array}{l}\text { RJD } \\
\text { days }\end{array}$ & $\begin{array}{c}S_{3 \mathrm{~mm}} \\
\mathrm{Jy}\end{array}$ & $\begin{array}{c}p_{3 \mathrm{~mm}} \\
\%\end{array}$ & $\chi_{\substack{3 \mathrm{~mm} \\
0}}$ \\
\hline 2006-09-24 & 54003 & $0.92 \pm 0.05$ & $15.6 \pm 0.5$ & $39.0 \pm 1.0$ \\
\hline 2006-10-03 & 54012 & $1.03 \pm 0.05$ & $13.4 \pm 0.4$ & $40.0 \pm 1.1$ \\
\hline $2006-10-13$ & 54022 & $0.90 \pm 0.05$ & $12.2 \pm 0.4$ & $39.0 \pm 1.6$ \\
\hline $2006-10-14$ & 54023 & $0.94 \pm 0.05$ & $14.4 \pm 2.1$ & $40.0 \pm 2.6$ \\
\hline $2006-11-16$ & 54056 & $0.87 \pm 0.04$ & $12.8 \pm 1.1$ & $32.1 \pm 1.9$ \\
\hline 2006-11-17 & 54057 & $0.85 \pm 0.04$ & $16.6 \pm 0.6$ & $39.5 \pm 0.9$ \\
\hline $2006-11-22$ & 54062 & $0.90 \pm 0.04$ & $13.9 \pm 0.5$ & $41.8 \pm 2.2$ \\
\hline 2006-12-01 & 54071 & $0.92 \pm 0.05$ & $14.1 \pm 0.8$ & $37.1 \pm 1.1$ \\
\hline 2007-01-03 & 54104 & $0.96 \pm 0.05$ & $14.0 \pm 1.4$ & $38.4 \pm 1.5$ \\
\hline 2007-01-17 & 54119 & $0.97 \pm 0.05$ & $14.9 \pm 0.4$ & $41.9 \pm 1.0$ \\
\hline $2007-01-29$ & 54130 & $0.94 \pm 0.05$ & $13.4 \pm 0.4$ & $36.1 \pm 1.0$ \\
\hline 2007-06-11 & 54262 & $0.83 \pm 0.04$ & $14.8 \pm 0.7$ & $31.1 \pm 1.3$ \\
\hline $2007-08-07$ & 54320 & $1.00 \pm 0.05$ & & \\
\hline $2007-10-09$ & 54383 & $0.85 \pm 0.04$ & $15.6 \pm 0.4$ & $37.4 \pm 1.0$ \\
\hline 2008-05-04 & 54591 & $0.87 \pm 0.06$ & $13.3 \pm 0.8$ & $30.1 \pm 1.9$ \\
\hline $2008-05-13$ & 54600 & $0.95 \pm 0.05$ & & \\
\hline $2008-12-29$ & 54830 & $1.02 \pm 0.05$ & $13.5 \pm 0.4$ & $39.1 \pm 1.1$ \\
\hline 2009-06-18 & 55001 & $0.87 \pm 0.04$ & $13.0 \pm 0.3$ & $39.4 \pm 0.8$ \\
\hline 2009-07-30 & 55043 & $0.90 \pm 0.04$ & $14.9 \pm 1.1$ & $38.9 \pm 1.3$ \\
\hline $2009-08-18$ & 55062 & $0.90 \pm 0.04$ & $13.9 \pm 0.4$ & $36.6 \pm 2.1$ \\
\hline 2010-05-05 & 55322 & $0.98 \pm 0.05$ & $13.6 \pm 0.4$ & $37.5 \pm 2.4$ \\
\hline 2010-06-06 & 55354 & $0.94 \pm 0.05$ & $12.6 \pm 0.3$ & $37.3 \pm 1.1$ \\
\hline 2010-06-16 & 55364 & $0.83 \pm 0.04$ & $13.5 \pm 0.3$ & $37.5 \pm 0.7$ \\
\hline 2010-08-15 & 55424 & $0.83 \pm 0.04$ & $15.4 \pm 0.3$ & $38.4 \pm 0.7$ \\
\hline $2010-10-12$ & 55482 & $0.93 \pm 0.05$ & $13.0 \pm 0.3$ & $33.8 \pm 0.8$ \\
\hline 2011-01-31 & 55593 & $0.87 \pm 0.04$ & $13.4 \pm 0.3$ & $33.0 \pm 0.7$ \\
\hline 2011-02-07 & 55600 & $0.95 \pm 0.05$ & $13.5 \pm 0.3$ & $35.2 \pm 0.7$ \\
\hline $2011-03-25$ & 55646 & $0.94 \pm 0.05$ & $13.5 \pm 0.2$ & $38.5 \pm 0.7$ \\
\hline 2011-04-10 & 55662 & $0.92 \pm 0.05$ & $13.4 \pm 0.3$ & $37.6 \pm 0.7$ \\
\hline 2011-04-25 & 55677 & $0.90 \pm 0.04$ & $12.1 \pm 0.3$ & $39.4 \pm 0.9$ \\
\hline 2011-05-04 & 55686 & $0.96 \pm 0.05$ & $13.2 \pm 0.3$ & $35.7 \pm 0.7$ \\
\hline $2011-05-22$ & 55704 & $0.89 \pm 0.04$ & $14.2 \pm 0.3$ & $36.2 \pm 0.7$ \\
\hline $2011-05-24$ & 55706 & $0.98 \pm 0.05$ & $15.1 \pm 0.3$ & $41.1 \pm 0.6$ \\
\hline 2011-06-15 & 55728 & $0.74 \pm 0.04$ & $13.1 \pm 0.3$ & $37.9 \pm 0.8$ \\
\hline 2011-09-04 & 55809 & $0.82 \pm 0.04$ & $13.6 \pm 0.3$ & $35.9 \pm 0.8$ \\
\hline 2011-09-11 & 55816 & $0.87 \pm 0.04$ & $12.6 \pm 0.3$ & $40.0 \pm 0.8$ \\
\hline 2011-10-11 & 55846 & $0.95 \pm 0.05$ & $12.2 \pm 0.3$ & $38.0 \pm 0.8$ \\
\hline 2011-10-16 & 55882 & $0.95 \pm 0.05$ & $13.8 \pm 0.3$ & $34.5 \pm 0.7$ \\
\hline 2012-01-03 & 55930 & $0.97 \pm 0.05$ & $13.3 \pm 0.3$ & $37.4 \pm 0.7$ \\
\hline $2012-01-30$ & 55957 & $1.02 \pm 0.05$ & $13.2 \pm 0.2$ & $37.4 \pm 0.7$ \\
\hline
\end{tabular}

The measurements performed before Spring 2009 used the orthogonal linearly polarized A100 and B100 heterodyne receivers tuned at $3 \mathrm{~mm}(86 \mathrm{GHz})$. After Spring 2009, we employed the new E090 and E230 pairs of orthogonal linearly polarized receivers at the IRAM $30 \mathrm{~m}$ Telescope to observe simultaneously at $3 \mathrm{~mm}$ and $1 \mathrm{~mm}(229 \mathrm{GHz})$, where the angular resolution of the telescope (full width at half power) is $28^{\prime \prime}$ and $11^{\prime \prime}$, respectively.

After a cross-scan pointing of the telescope and an amplitude, and phase calibration measurement (see Thum et al. 2008), every XPOL measurement consisting of wobbler switching onoffs was performed for a total integration time $\sim 8 \mathrm{~min}$. The signal from each of the two orthogonal $3 \mathrm{~mm}$ receivers was connected to the XPOL polarimeter, whose output consisted of a $640 \mathrm{MHz}$ spectrum from each of the receivers, plus the real and imaginary part of their cross-correlation. These four XPOL observables were used to compute the four Stokes parameters for each measurement (Thum et al. 2008). The XPOL hardware limitations only allow one to connect $320 \mathrm{MHz}$ to the remaining bandwidth of the polarimeter. This bandwidth was used for the simultaneous $1 \mathrm{~mm}$ observations taken after the end of 2009.
Table 2. $1 \mathrm{~mm}$ photo-polarimetric data from 3C 286.

\begin{tabular}{lcccc}
\hline \hline Date & $\begin{array}{c}\text { RJD } \\
\text { days }\end{array}$ & $\begin{array}{c}S_{1 \mathrm{~mm}} \\
\mathrm{Jy}\end{array}$ & $\begin{array}{c}p_{1 \mathrm{~mm}} \\
\%\end{array}$ & $\begin{array}{c}\chi_{1 \mathrm{~mm}} \\
{ }^{\circ}\end{array}$ \\
\hline $2010-05-05$ & 55322 & $0.40 \pm 0.02$ & $14.3 \pm 2.1$ & $38.6 \pm 3.9$ \\
$2010-06-06$ & 55354 & $0.33 \pm 0.02$ & $\ldots$ & $\ldots$ \\
$2010-10-12$ & 55482 & $0.34 \pm 0.03$ & $\ldots$ & $\ldots$ \\
$2011-01-31$ & 55593 & $0.31 \pm 0.02$ & $18.0 \pm 2.4$ & $22.3 \pm 3.9$ \\
$2011-02-07$ & 55600 & $0.34 \pm 0.02$ & $13.9 \pm 2.5$ & $30.8 \pm 5.0$ \\
$2011-03-25$ & 55646 & $0.28 \pm 0.02$ & $17.7 \pm 3.4$ & $35.8 \pm 6.2$ \\
$2011-04-10$ & 55662 & $0.23 \pm 0.01$ & $17.5 \pm 3.2$ & $23.7 \pm 5.2$ \\
$2011-04-25$ & 55677 & $0.21 \pm 0.02$ & $13.4 \pm 5.5$ & $19.3 \pm 11.9$ \\
$2011-05-04$ & 55686 & $0.28 \pm 0.02$ & $18.5 \pm 2.8$ & $41.6 \pm 4.0$ \\
$2011-09-11$ & 55816 & $0.26 \pm 0.02$ & $13.9 \pm 4.7$ & $38.1 \pm 9.7$ \\
$2011-10-11$ & 55846 & $0.31 \pm 0.02$ & $17.0 \pm 5.8$ & $47.9 \pm 8.6$ \\
$2011-10-16$ & 55882 & $0.34 \pm 0.02$ & $14.1 \pm 2.5$ & $32.2 \pm 5.1$ \\
$2012-01-03$ & 55930 & $0.39 \pm 0.02$ & $10.2 \pm 1.8$ & $47.1 \pm 5.4$ \\
$2012-01-30$ & 55957 & $0.36 \pm 0.02$ & $13.2 \pm 2.1$ & $23.8 \pm 4.6$ \\
\hline
\end{tabular}

After calibrating the amplitudes and phases from every pair of linearly polarized orthogonal receivers, the instrumental polarization for every observing epoch (estimated through measurements of unpolarized calibrators, i.e. Mars and/or Uranus, when available) was removed from the data. The instrumental polarization parameters used for the $3 \mathrm{~mm}$ observations before Spring 2009 were $Q_{\mathrm{i}} \approx 0.005 I, U_{\mathrm{i}} \approx 0.003 I$, for the contribution of the instrumental polarization to the $Q$ and $U$ Stokes parameters, respectively. After mid-2009, new instrumental polarization parameters characterized the performance of the new E090 and E230 receiver pairs on the $30 \mathrm{~m}$ Telescope. These are $Q_{\mathrm{i}} \approx-0.007 I$ and $U_{\mathrm{i}} \approx-0.003 I$ for $3 \mathrm{~mm}$ observations and $Q_{\mathrm{i}} \approx-0.015 \mathrm{I}$ and $U_{\mathrm{i}} \approx-0.015 I$ for $1 \mathrm{~mm}$. The Jy/K calibration factor $\left(C_{\mathrm{Jy} / \mathrm{K}}\right)$ for the total flux density scales were computed for every observing epoch before Spring 2009 as in Agudo et al. (2006). The resulting $C_{\mathrm{Jy} / \mathrm{K}}$ values were fully consistent with the standard $C_{\mathrm{Jy} / \mathrm{K}}$ calibration factors at 3 and $1 \mathrm{~mm}$ for the IRAM $30 \mathrm{~m}$ Telescope (6.4 Jy/K and $9.3 \mathrm{Jy} / \mathrm{K}$, respectively; Ungerechts, priv. comm.). For the epochs after mid-2009 we used these standard $C_{\mathrm{Jy} / \mathrm{K}}$ calibration factors.

The total flux density uncertainties given in Tables 1 and 2 correspond to the statistical uncertainties for every specific measurement plus a 5\% systematic factor from the uncertainties in $C_{\mathrm{Jy} / \mathrm{K}}$ (Agudo et al. 2006) that was added quadratically. The linear polarization degree $(p)$ and linear polarization angle $(\chi)$ uncertainties in Tables 1 and 2 were estimated from the statistical uncertainties from every measurement, plus a non-systematic contribution computed from the dispersion of $Q$, and $U$ Stokes parameters from measurements of Mars and Uranus. These dispersion estimates are $\Delta Q_{\mathrm{i}}=0.003 \mathrm{I}$, and $\Delta U_{\mathrm{i}}=0.002 \mathrm{I}$, for both the 3 and $1 \mathrm{~mm}$ observations. These give polarizationuncertainty medians of $\Delta p \approx 0.5 \%, \Delta \chi \approx 1^{\circ}$ at $3 \mathrm{~mm}$, and $\Delta p \approx 4 \%, \Delta \chi \approx 5^{\circ}$ at $1 \mathrm{~mm}$ for our measurements of $3 \mathrm{C} 286$. The absolute calibration of the polarization angle of the XPOL polarimeter has a precision of $0.5^{\circ}$ or better (Thum et al. 2008; Aumont et al. 2010). Hence, we did not consider a significant source of error from this calibration on the uncertainties of our final measurements.

\section{Results and discussion}

\section{1. $3 \mathrm{~mm}$}

Figure 1 shows the $3 \mathrm{~mm}$ total flux density $\left(S_{3 \mathrm{~mm}}\right)$, linear polarization degree $\left(p_{3 \mathrm{~mm}}\right)$, and polarization angle $\left(\chi_{3 \mathrm{~mm}}\right)$ evolution 
Table 3. Best-fit linear model parameters for $S_{3 \mathrm{~mm}}(t), p_{3 \mathrm{~mm}}(t)$, and $\chi_{3 \mathrm{~mm}}(t)$.

\begin{tabular}{|c|c|c|c|c|c|c|}
\hline $\begin{array}{l}\text { Par. } \\
\text { (1) }\end{array}$ & $\begin{array}{l}\text { Est. } \\
(2)\end{array}$ & $\begin{array}{c}\epsilon \\
(3) \\
\end{array}$ & $\begin{array}{c}t \text {-stat } \\
\text { (4) }\end{array}$ & $\begin{array}{c}P \\
(5) \\
\end{array}$ & $\begin{array}{l}95 \% \mathrm{C} \\
(6)\end{array}$ & $\begin{array}{r}\text { ff. Int. } \\
\text { (7) }\end{array}$ \\
\hline \multicolumn{7}{|c|}{$S_{3 \mathrm{~mm}}$} \\
\hline$a$ & 0.915 & 0.018 & 51 & $1.1 \times 10^{-36}$ & 0.879 & 0.951 \\
\hline$b$ & $-7 \times 10^{-6}$ & $1.4 \times 10^{-5}$ & -0.5 & 0.62 & $-4 \times 10^{-5}$ & $2.2 \times 10^{-5}$ \\
\hline \multicolumn{7}{|c|}{$p_{3 \mathrm{~mm}}$} \\
\hline$a$ & 14.18 & 0.36 & 39 & $4 \times 10^{-31}$ & 13.45 & 14.91 \\
\hline$b$ & $-5 \times 10^{-4}$ & $2.4 \times 10^{-4}$ & -1.9 & 0.06 & $-9 \times 10^{-4}$ & $3 \times 10^{-5}$ \\
\hline \multicolumn{7}{|c|}{$\chi_{3 \mathrm{~mm}}$} \\
\hline$a$ & 38.19 & 0.88 & 43 & $1.1 \times 10^{-32}$ & 36.41 & 39.98 \\
\hline$b$ & $-6 \times 10^{-4}$ & $6 \times 10^{-4}$ & -1.07 & 0.29 & $-1.8 \times 10^{-3}$ & $6 \times 10^{-4}$ \\
\hline
\end{tabular}

Notes. Columns are as follows: (1) fitted parameter, (2) estimate from the fit, (3) standard error, (4) $t$-statistic, (5) its corresponding $P$-value, and (6) and (7) lower and upper limits of the $95 \%$ confidence interval, respectively.

of 3C 286 as measured from mid 2006 to the beginning of 2012 (see also Table 1). Neither $S_{3 \mathrm{~mm}}$ nor $p_{3 \mathrm{~mm}}$ and $\chi_{3 \mathrm{~mm}}$ show appreciable variability during the time range of our observations. Indeed, Fig. 1 resembles the behavior of a non-varying total flux and polarization source, as observed for 3C 286 at longer centimeter wavelengths.

To verify the stability of $S_{3 \mathrm{~mm}}, p_{3 \mathrm{~mm}}$, and $\chi_{3 \mathrm{~mm}}$ with time, we tested if these variables can be fitted by a constant. Initially we fitted a linear model to each of these data sets. This model is characterized by a slope $(b)$ and an intercept $(a)$, i.e. $y=a+b t$, were $t$ is the time and $y$ is either $S_{3 \mathrm{~mm}}, p_{3 \mathrm{~mm}}$, or $\chi_{3 \mathrm{~mm}}$. The resulting linear-model best-fit parameters are given in Table 3 . We tested the hypothesis that the slope $b$ is equal to 0 (which we took as the Null Hypothesis, $H_{0}$ ). In Table 3 we show that for $b$, the $P$-values from the $t$-statistic are $62 \%, 6 \%$, and $29 \%$ for $S_{3 \mathrm{~mm}}$, $p_{3 \mathrm{~mm}}$, and $\chi_{3 \mathrm{~mm}}$ respectively. This shows that the probabilities of obtaining the fitted values of $b$ by chance are not small. Based on the values of the $t$-statistic, we also estimated the $95 \%$ confidence intervals for the fitted values of $b$ (see Table 3 ). The zerovalue (i.e. $b=0$ ) lies within the $95 \%$ confidence interval for $S_{3 \mathrm{~mm}}(t), p_{3 \mathrm{~mm}}(t)$, and $\chi_{3 \mathrm{~mm}}(t)$. Since the $P$-values are greater than the $5 \%$ significance level, the data do not provide grounds for rejecting the $H_{0}$ at a $5 \%$ significance level. Therefore, the measurements of $S_{3 \mathrm{~mm}}, p_{3 \mathrm{~mm}}$, and $\chi_{3 \mathrm{~mm}}$ are distributed around a constant value, within a $95 \%$ confidence level.

Fitting a constant model (i.e., $y=a$ ) to the data additionally verifies the above picture (see also Fig. 1). This is demonstrated by the remarkably low $P$-values for obtaining the fitted value by chance (see Table 4). The resulting fitted constants of $S_{3 \mathrm{~mm}}$, $p_{3 \mathrm{~mm}}$, and $\chi_{3 \mathrm{~mm}}$ for $3 \mathrm{C} 286$, and their $95 \%$ confidence intervals are $S_{3 \mathrm{~mm}}=(0.91 \pm 0.02) \mathrm{Jy}, p_{3 \mathrm{~mm}}=(13.5 \pm 0.3) \%$, and $\chi_{3 \mathrm{~mm}}=$ $(37.3 \pm 0.8)^{\circ}$ (see Table 4 and Fig. 1). We propose these as the values of $S_{3 \mathrm{~mm}}, p_{3 \mathrm{~mm}}$, and $\chi_{3 \mathrm{~mm}}$ to use for calibration through observations of $3 \mathrm{C} 286$.

\section{2. $1 \mathrm{~mm}$}

At $1 \mathrm{~mm}$ (Fig. 2 and Table 2), the shorter observing bandwidth, the poorer atmospheric transmission and receiver sensitivity and the weakness of the source at this wavelength together with the poorer time coverage at this spectral range in our monitoring of 3C 286 do not allow us to easily discern the stable $S_{1 \mathrm{~mm}}, p_{1 \mathrm{~mm}}$, and $\chi_{1 \mathrm{~mm}}$ pattern seen at $3 \mathrm{~mm}$ by visual inspection. However, for the linear fit of $S_{1 \mathrm{~mm}}(t), p_{1 \mathrm{~mm}}(t)$, and $\chi_{1 \mathrm{~mm}}(t)$ (Table 5), the probabilities of obtaining the fitted values of $b$ by chance are
Table 4. Best-fit constant model parameters for $S_{3 \mathrm{~mm}}(t), p_{3 \mathrm{~mm}}(t)$, and $\chi_{3 \mathrm{~mm}}(t)$.

\begin{tabular}{|c|c|c|c|c|c|}
\hline $\begin{array}{l}a \\
(1)\end{array}$ & $\begin{array}{c}\epsilon \\
(2)\end{array}$ & $\begin{array}{c}t \text {-stat } \\
\text { (3) }\end{array}$ & $\begin{array}{c}P \\
(4)\end{array}$ & $\begin{array}{c}95 \% \mathrm{C} \\
(5)\end{array}$ & $\begin{array}{l}\text { nf. Int. } \\
\text { (6) }\end{array}$ \\
\hline 0.908 & 0.010 & 89 & $\begin{array}{l}S_{3 \mathrm{~mm}} \\
1.2 \times 10^{-46}\end{array}$ & 0.887 & 0.928 \\
\hline 13.54 & 0.15 & 93 & $\begin{array}{l}p_{3 \mathrm{~mm}} \\
2.2 \times 10^{-45}\end{array}$ & 13.25 & 13.84 \\
\hline 37.35 & 0.38 & 97 & $\begin{array}{l}\chi_{3 \mathrm{~mm}} \\
3 \times 10^{-46}\end{array}$ & 36.57 & 38.13 \\
\hline
\end{tabular}

Notes. Columns are as follows: (1) estimate of $a$ from the fit, (2) standard error, (3) $t$-statistic, (4) its corresponding $P$-value, and (5) and (6) lower and upper limits of the $95 \%$ confidence interval, respectively.

also fairly high; $99 \%, 12 \%$ and $94 \%$, respectively. The $95 \%$ confidence intervals for the fitted values of $b$ for $S_{1 \mathrm{~mm}}(t), p_{1 \mathrm{~mm}}(t)$, and $\chi_{1 \mathrm{~mm}}(t)$, also include $b=0$. The corresponding $P$-values are greater than the $5 \%$ significance level. Thus, we can also confirm, within a $95 \%$ confidence level, that the measurements of $S_{1 \mathrm{~mm}}, p_{1 \mathrm{~mm}}$, and $\chi_{1 \mathrm{~mm}}$ are distributed around a constant value. This is confirmed by the constant fits to the $1 \mathrm{~mm}$ data sets. Their expected values and $95 \%$ confidence intervals are $S_{1 \mathrm{~mm}}=$ $(0.30 \pm 0.03) \%, p_{1 \mathrm{~mm}}=(14.4 \pm 1.8) \%$, and $\chi_{1 \mathrm{~mm}}=(33.1 \pm 5.7)^{\circ}$ (Table 6 and Fig. 2).

\subsection{Total flux and polarization properties of $3 C 286$ along the radio and millimeter spectrum}

Figure 3 and Table 7 show the total flux density and linear polarization properties of 3C 286 along the millimeter and radiocentimeter spectrum (from $1 \mathrm{~mm}$ to $21 \mathrm{~cm}$ ). The corresponding data were taken either from the results presented here, or from Peng et al. (2000) and Taylor et al. (2001), see Table 7.

The flux density plot shows a monotonically decreasing spectral trend as expected for optically-thin synchrotron emission from relativistic jets (e.g., Marscher \& Gear 1985). Despite some moderated spectral-index jumps seen for the measurements with larger errors (Fig. 4 and Table 8), the spectral index also shows an overall decreasing pattern along the broad radiomillimeter spectral-range considered here. No evident spectral break is observed from Fig. 3, which suggests that the synchrotron radiation observed from 3C 286 at $1 \mathrm{~mm}$ through $21 \mathrm{~cm}$ was produced by a single-electron population. 
Table 5. Same as Table 3 but for the $1 \mathrm{~mm}$ data.

\begin{tabular}{|c|c|c|c|c|c|c|}
\hline $\begin{array}{l}\text { Par. } \\
(1)\end{array}$ & $\begin{array}{l}\text { Est. } \\
(2)\end{array}$ & $\begin{array}{c}\epsilon \\
(3) \\
\end{array}$ & $\begin{array}{c}t \text {-stat } \\
(4)\end{array}$ & $\begin{array}{c}P \\
(5) \\
\end{array}$ & \multicolumn{2}{|c|}{ 95\% Conf. Int. } \\
\hline \multicolumn{7}{|c|}{$S_{1 \mathrm{~mm}}$} \\
\hline $\begin{array}{l}a \\
b \\
\end{array}$ & $\begin{array}{c}0.303 \\
-8 \times 10^{-7} \\
\end{array}$ & $\begin{array}{c}0.120 \\
8 \times 10^{-5} \\
\end{array}$ & $\begin{array}{r}2.5 \\
-0.009 \\
\end{array}$ & $\begin{array}{c}2.4 \times 10^{-2} \\
0.99 \\
\end{array}$ & $\begin{array}{c}0.046 \\
-1.8 \times 10^{-4} \\
\end{array}$ & $\begin{array}{c}0.560 \\
1.8 \times 10^{-4} \\
\end{array}$ \\
\hline \multicolumn{7}{|c|}{$p_{1 \mathrm{~mm}}$} \\
\hline $\begin{array}{l}a \\
b \\
\end{array}$ & $\begin{array}{c}16.84 \\
-6 \times 10^{-3} \\
\end{array}$ & $\begin{array}{c}1.61 \\
4 \times 10^{-3} \\
\end{array}$ & $\begin{array}{c}10 \\
-1.7 \\
\end{array}$ & $\begin{array}{c}1.1 \times 10^{-6} \\
0.12 \\
\end{array}$ & $\begin{array}{c}13.25 \\
-1.4 \times 10^{-2} \\
\end{array}$ & $\begin{array}{c}20.43 \\
2.0 \times 10^{-3} \\
\end{array}$ \\
\hline \multicolumn{7}{|c|}{$\chi_{1 \mathrm{~mm}}$} \\
\hline $\begin{array}{l}a \\
b\end{array}$ & $\begin{array}{c}33.46 \\
-1 \times 10^{-3}\end{array}$ & $\begin{array}{c}5.69 \\
0.014 \\
\end{array}$ & $\begin{array}{c}6 \\
-0.08 \\
\end{array}$ & $\begin{array}{c}1.6 \times 10^{-4} \\
0.94\end{array}$ & $\begin{array}{r}20.78 \\
-0.03 \\
\end{array}$ & $\begin{array}{c}46.13 \\
0.03 \\
\end{array}$ \\
\hline
\end{tabular}

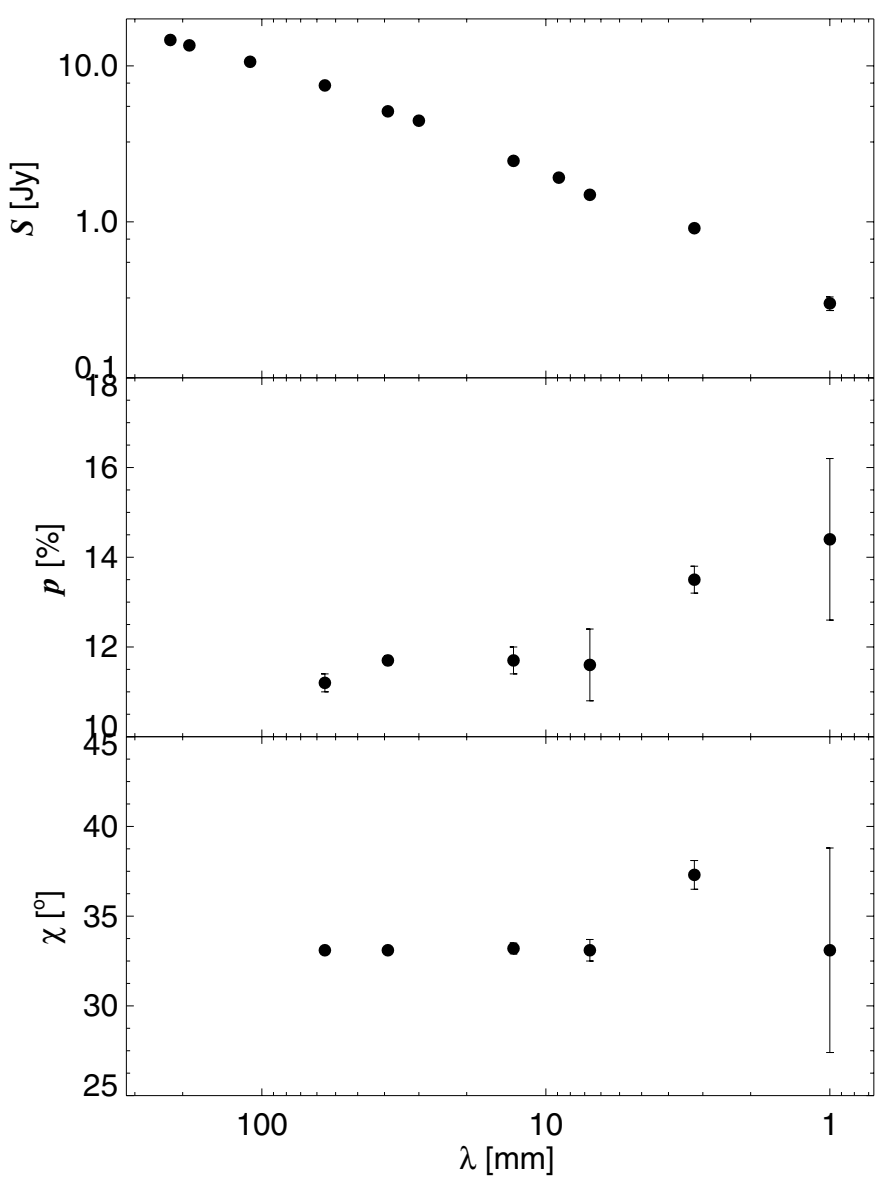

Fig. 3. Total flux density, polarization degree, and polarization angle of 3C 286 along the radio and millimeter spectrum. Plotted data are those presented in Table 7.

The $3 \mathrm{~mm}$ and $1 \mathrm{~mm}$ polarization properties of 3C 286 shown in Fig. 3 seem to be quite similar to those at longer wavelengths within a $\sim 2 \%$ fractional polarization and $\sim 4^{\circ}$ polarization angle. However, the $3 \mathrm{~mm}$ and $1 \mathrm{~mm}$ polarization degree ( $p_{3 \mathrm{~mm}} \approx 13.5 \%$ and $p_{1 \mathrm{~mm}} \approx 14.5 \%$, respectively) seem to increase slightly for decreasing wavelengths compared to the fractional polarization at wavelengths longer than $3 \mathrm{~mm}$ (that lie at the $[11,12] \%$ level). The polarization angle that we measure at $3 \mathrm{~mm}\left(\chi_{3 \mathrm{~mm}} \approx 37^{\circ}\right)$ is also moderately different from the one measured at longer wavelengths $\left(\sim 33^{\circ}\right.$, see Fig. 3 and Table 7$)$. Our $1 \mathrm{~mm}$ polarization angle measurement $\left(\chi_{1 \mathrm{~mm}}=33 \pm 6^{\circ}\right)$ has sufficiently large uncertainty to avoid discerning if the shorter

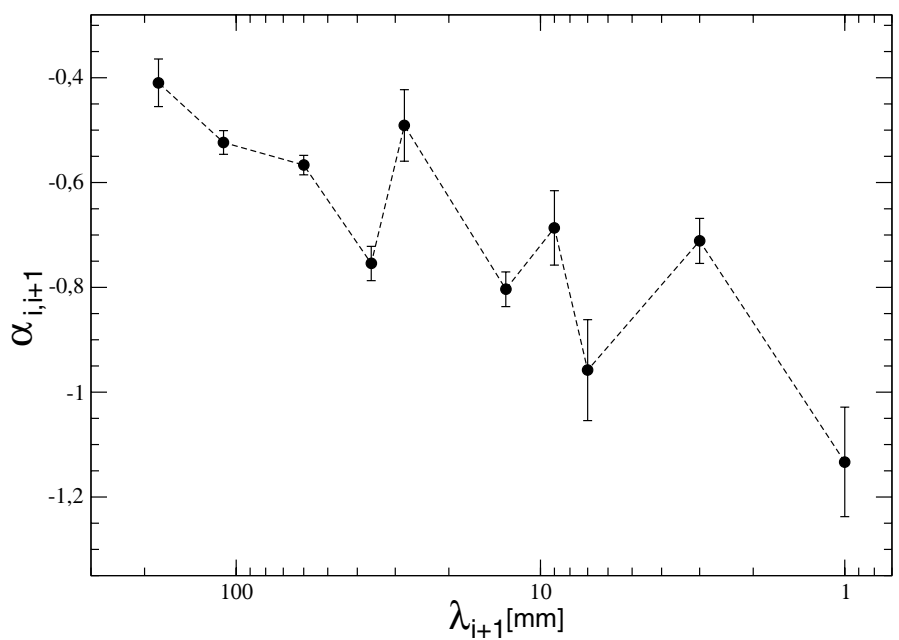

Fig. 4. Spectral index of $3 \mathrm{C} 286$ as a function of $v_{\mathrm{i}+1}$. The data presented here are those from Table 8.

Table 6. Same as Table 4 but for the $1 \mathrm{~mm}$ data.

\begin{tabular}{|c|c|c|c|c|c|}
\hline $\begin{array}{l}a \\
(1)\end{array}$ & $\begin{array}{c}\epsilon \\
(2)\end{array}$ & $\begin{array}{c}t \text {-stat } \\
\text { (3) }\end{array}$ & $\begin{array}{c}P \\
(4)\end{array}$ & $\begin{array}{c}95 \% \\
(5)\end{array}$ & $\begin{array}{l}\text { inf. Int. } \\
(6)\end{array}$ \\
\hline 0.302 & 0.014 & 21 & $\begin{array}{l}S_{1 \mathrm{~mm}} \\
1.2 \times 10^{-12}\end{array}$ & 0.272 & 0.332 \\
\hline 14.44 & 0.82 & 18 & $\begin{array}{l}p_{1 \mathrm{~mm}} \\
2.0 \times 10^{-9}\end{array}$ & 12.64 & 16.24 \\
\hline 33.07 & 2.60 & 13 & $\begin{array}{l}\chi_{1 \mathrm{~mm}} \\
\quad 6 \times 10^{-8} \\
\end{array}$ & 27.35 & 38.80 \\
\hline
\end{tabular}

wavelength polarization angle matches the values of the $3 \mathrm{~mm}$ measurements or those at longer wavelengths. We hope to solve this ambiguity by better constraining the $1 \mathrm{~mm}$ polarization angle of 3C 286 through the continued monitoring of the source, which we currently perform at the IRAM $30 \mathrm{~m}$ Telescope.

The slightly different fractional polarization and polarization angle at the shorter wavelengths in 3C 286 can be explained if the fraction of tangled magnetic field decreases toward inner regions upstream in the jet. Some fraction of this tangled magnetic field is needed to explain the decreased fractional polarization observed in the entire radio and millimeter spectrum compared to the predicted value for optically thin polarized synchrotron-radiation ( 70\%, Pacholczyk 1970), if Faraday depolarization is negligible (which is clearly the case for 3C 286 , see Fig. 3). Moreover, the bulk jet emission should be radiated from jet regions at inner upstream locations for shorter wavelengths, as is usually observed through very long baseline 
Table 7. $S, p$, and $\chi$ measurements of 3C 286 along the millimeter and radio spectrum.

\begin{tabular}{lcccc}
\hline \hline$\lambda$ & $S$ & $p$ & $\chi$ & Ref. \\
$\mathrm{mm}$ & $\mathrm{Jy}$ & $\begin{array}{c}p \\
{ }^{a}\end{array}$ & \\
\hline $1^{a}$ & $0.30 \pm 0.03$ & $14.4 \pm 1.8$ & $33.1 \pm 5.7$ & 1 \\
$3^{a}$ & $0.91 \pm 0.02$ & $13.5 \pm 0.3$ & $37.3 \pm 0.8$ & 1 \\
7 & $1.49 \pm 0.03^{b}$ & $11.6 \pm 0.8^{c}$ & $33.1 \pm 0.6^{c}$ & 2,3 \\
9 & $1.92 \pm 0.03^{b}$ & $\ldots$ & $\ldots$ & 2 \\
13 & $2.46 \pm 0.05^{b}$ & $11.7 \pm 0.3^{c}$ & $33.2 \pm 0.3^{c}$ & 2,3 \\
28 & $4.45 \pm 0.06^{b}$ & $\ldots$ & $\ldots$ & 2 \\
36 & $5.11 \pm 0.07^{b}$ & $11.7 \pm 0.1^{c}$ & $33.1 \pm 0.1^{c}$ & 2,3 \\
60 & $7.49 \pm 0.07^{b}$ & $11.2 \pm 0.2^{c}$ & $33.1 \pm 0.2^{c}$ & 2,3 \\
110 & $10.62 \pm 0.07^{b}$ & $\ldots$ & $\ldots$ & 2 \\
180 & $13.53 \pm 0.11^{b}$ & $\ldots$ & $\ldots$ & 2 \\
210 & $14.65 \pm 0.05^{b}$ & $\ldots$ & $\ldots$ & 2 \\
\hline
\end{tabular}

Notes. ${ }^{(a)} S, p$, and $\chi$ correspond to fitted values as presented in this work. ${ }^{(b)}$ Average values of $S$ are those presented by Peng et al. (2000). ${ }^{(c)}$ Average values of measurements made during 2009 by Taylor et al. (2001), see http://www.vla.nrao.edu/astro/calib/ polar/2009/.

References. (1) This paper; (2) Peng et al. (2000); (3) Taylor et al. (2001).

interferometry in relativistic jets in active galactic nuclei (e.g., Sokolovsky et al. 2011). Within this scenario, the radiation emitted at shorter wavelengths from the inner jet region would show higher fractional polarization than the longer wavelength emission. The different polarization angles for radiation emitted from inner jet regions may be explained by a slightly different jet direction compared to the jet section where the longer wavelength emission is radiated (e.g., Abdo et al. 2010). This can be easily accommodated within Cotton et al.'s model for 3C 286 (see Fig. 6 in Cotton et al. 1997), which uses a bend in the inner jet regions to explain the apparent lack of a high brightness temperature core - beamed away from Earth in their model - as the cause for the unusual total flux and polarization properties of the source.

\section{Summary and conclusions}

We have shown that although 3C 286 displays moderate millimeter flux densities $\left(S_{3 \mathrm{~mm}} \approx 1 \mathrm{Jy}\right.$ and $\left.S_{1 \mathrm{~mm}} \approx 0.3 \mathrm{Jy}\right)$, its high millimeter polarization degree $\left(p_{3 \mathrm{~mm}} \approx 13.5 \%\right.$ and $\left.p_{1 \mathrm{~mm}} \approx 14.5 \%\right)$ and its time stability make this source a valuable polarization calibrator for millimeter observations with instruments sensitive enough to detect linear polarization as bright as $\sim 120 \mathrm{mJy}$ at $3 \mathrm{~mm}$ and $\sim 45 \mathrm{mJy}$ at $1 \mathrm{~mm}$.

The $3 \mathrm{~mm}$ and $1 \mathrm{~mm}$ total flux and polarization properties of 3C 286 were shown to be fairly similar to those previously known at longer wavelengths, which additionally supports the suitability of extending the use of 3C 286 as total flux and polarization calibrator from radio to short millimeter wavelengths. The slightly higher $3 \mathrm{~mm}$ and $1 \mathrm{~mm}$ fractional polarization of the source compared to those at longer wavelengths $(p \in[11,12] \%$ from $7 \mathrm{~mm}$ to $6 \mathrm{~cm}$ ), can be explained if the degree of tangled magnetic field in the jet decreases toward inner upstream jet regions. To explain the deviation of $\sim 4^{\circ}$ of the $3 \mathrm{~mm}$ (and perhaps $1 \mathrm{~mm}$ ) polarization angle compared to that at longer wavelengths, a small bend in the inner jet regions is also needed, but this is fully consistent with previous models for 3C 286. The time-dependent behavior of the source at $3 \mathrm{~mm}$ and $1 \mathrm{~mm}$ was shown to be as stable as is expected at shorter millimeter and
Table 8. Spectral indexes of flux densities for contiguous wavelenghts as shown in Table 7.

\begin{tabular}{lcccc}
\hline \hline $\begin{array}{l}\lambda_{i+1} \\
\mathrm{~mm}\end{array}$ & $\begin{array}{c}v_{i+1} \\
\mathrm{GHz}\end{array}$ & $\begin{array}{c}\lambda_{i} \\
\mathrm{~mm}\end{array}$ & $\begin{array}{c}v_{i+1} \\
\mathrm{GHz}\end{array}$ & $\alpha_{i, i+1}$ \\
\hline 1 & 229 & 3 & 86 & $-1.13 \pm 0.11$ \\
3 & 86 & 7 & 43 & $-0.71 \pm 0.04$ \\
7 & 43 & 9 & 33 & $-0.96 \pm 0.10$ \\
9 & 33 & 13 & 22 & $-0.69 \pm 0.07$ \\
13 & 22 & 28 & 11 & $-0.80 \pm 0.03$ \\
28 & 11 & 36 & 8.4 & $-0.49 \pm 0.07$ \\
36 & 8.4 & 60 & 5 & $-0.75 \pm 0.03$ \\
60 & 5 & 110 & 2.7 & $-0.57 \pm 0.02$ \\
110 & 2.7 & 180 & 1.7 & $-0.52 \pm 0.02$ \\
180 & 1.7 & 210 & 1.4 & $-0.41 \pm 0.05$ \\
\hline
\end{tabular}

Notes. The spectral index is defined such that $\alpha_{i, i+1}=\frac{\log \left(S_{\mathrm{i}} / S_{i+1}\right)}{\log \left(v_{i} / v_{i+1}\right)}$.

radio wavelengths, which also supports the suitability of 3C 286 for total flux and polarization calibration purposes.

Having 3C 286 as an additional polarization calibrator may be an advantage for calibration and/or observing programs with limited observing time if no other reliable polarization calibrator lies in the sky during their observing window. 3C 286 has a maximum extension of up to $\sim 3.5^{\prime \prime}$ in the plane of the sky. In contrast, other available polarization calibrators show extended structures as large as $\sim 5^{\prime}$, e.g. the Crab nebula (Aumont et al. 2010 ), or even $\sim 12^{\prime}$ for Cygnus A (Zemcov et al. 2010). Hence, 3C 286 may facilitate polarization calibration observations with high-resolution interferometers such as ALMA, Plateau de Bure, or the Sub-mm Array on extended configurations. Moreover, given the moderate northern declination of 3C 286 (see Sect. 1) it can be observed comfortably from all northern millimeter observatories, and (at elevations up to $\approx 30^{\circ}$ ) even from most southern millimeter observatories, including ALMA.

We conclude that 3C 286 may be safely used for calibration of both single-dish and interferometric polarization observations at $3 \mathrm{~mm}$, and possibly at shorter wavelengths.

Acknowledgements. The authors thank the anonymous referee for the constructive revision of this paper. The observations with the IRAM $30 \mathrm{~m}$ Telescope were made remotely from many different places. We thank the Observatory staff for their competent support. The IRAM $30 \mathrm{~m}$ Telescope is supported by INSU/CNRS (France), MPG (Germany), and IGN (Spain). This research has made use of the VLA/VLBA Polarization Calibration Database that was maintained by the National Radio Astronomy Observatory (NRAO) of the USA, until the end of 2009. The NRAO is a facility of the USA's National Science Foundation operated under cooperative agreement by USA's Associated Universities, Inc. This research was partly funded by the "Consejería de Economía, Innovación y Ciencia" of the Regional Government of Andalucía through grant P09-FQM-4784, an by the "Ministerio de Economía y Competitividad" of Spain through grant AYA2010-14844. D.E. acknowledges the Science and Technology Facilities Council (STFC) of the United Kingdom for support under grant ST/G003084/1.

\section{References}

Abdo, A. A., Ackermann, M., Ajello, M., et al. 2010, Nature, 463, 919 Agudo, I., Krichbaum, T. P., Ungerechts, H., et al. 2006, A\&A, 456, 117 Agudo, I., Thum, C., Wiesemeyer, H., \& Krichbaum, T. P. 2010, ApJS, 189, 1 Agudo, I., Jorstad, S. G., Marscher, A. P., et al. 2011a, ApJ, 726, L13 Agudo, I., Marscher, A. P., Jorstad, S. G., et al. 2011b, ApJ, 735, L10 Akujor, C. E., \& Garrington S. T. 1995, A\&AS, 112, 235

Aumont, J., Conversi, L., Thum, C., et al. 2010, A\&A, 514, A70

Baars, J. W. M., Genzel, R., Pauliny-Toth, I. I. K., \& Witzel, A. 1977, A\&A, 61, 99

Barvainis, R., Clemens, D. P., \& Leach, R. 1988, AJ, 95, 510

Burbidge, G. R., \& Burbidge, E. M. 1969, Nature, 224, 21

Cotton, W. D., Fanti, C., Fanti, R., et al. 1997, A\&A, 325, 479 
I. Agudo et al.: 3C 286: a polarization calibrator for millimeter observations

Fanti, C., Fanti, R., Parma, P., Schilizzi, R. T., \& van Breugel, W. J. M. 1985, A\&A, 143, 292

Gómez, J. L., Marscher, A. P., Alberdi, A., Jorstad, S. G., \& Agudo, I. 2002, VLBA Scientific Memorandum, 30, 1 http://www.vlba.nrao.edu/ memos/sci/sci30memo.ps

Jiang, D. R., Dallacasa, D., Schilizzi, R. T., et al. 1996, A\&A, 312, 380

Kraus, A., Quirrenbach, A., Lobanov, A. P., et al. 1999, A\&A, 344, 807

Marscher, A. P., \& Gear, W. K. 1985, ApJ, 298, 114

Matsumura, T., Ade, P., Barkats, D., et al. 2010, Proc. SPIE, 7741, 77412 O

McKinnon, M. M. 1992, A\&A, 260, 533

Ott, M., Witzel, A., Quirrenbach, A., et al. 1994, A\&A, 284, 331

Pacholczyk, A. G. 1970, Radio astrophysics. Nonthermal Processes in Galactic and Extragalactic Sources (San Francisco: Freeman)
Peacock, J. A., \& Wall, J. V. 1982, MNRAS, 198, 843

Peng, B., Kraus, A., Krichbaum, T. P., \& Witzel, A. 2000, A\&AS, 145, 1

Perley, R. A. 1982, AJ, 87, 859

Rudnick, L., \& Jones, T. W. 1983, AJ, 88, 518

Sokolovsky, K. V., Kovalev, Y. Y., Pushkarev, A. B., \& Lobanov, A. P. 2011, A\&A, 532, A38

Taylor, G. B., \& Myers, S. T. 2001, VLBA Scientific Memorandum, 26, 1 http://www.vlba.nrao.edu/memos/sci/sci26memo.ps

Thum, C., Wiesemeyer, H., Morris, D., Navarro, S., \& Torres, M. 2003, Proc. SPIE, 4843, 272

Thum, C., Wiesemeyer, H., Paubert, G., Navarro, S., \& Morris, D. 2008, PASP, 120,777

Zemcov, M., Ade, P., Bock, J., et al. 2010, ApJ, 710, 1541 